\title{
Hepatic Ganglioside Abnormalities in a Patient with Familial Erythrophagocytic Lymphohistiocytosis
}

\author{
CLIFFORD G. WONG, STEPHAN LADISCH, ${ }^{(56)}$ AND CHARLES C. SWEELEY \\ Division of Hematology-Oncology and Gwynne Hazen Cherry Memorial Laboratories, Department of Pediatrics, \\ UCLA School of Medicine, Los Angeles, California [C.G.W., S.L.] and Department of Biochemistry, Michigan State \\ University, E. Lansing, Michigan, USA [C.C.S.]
}

\begin{abstract}
Summary
Familial erythrophagocytic lymphohistiocytosis (FEL) is an autosomal recessive disease of unknown pathogenesis. Because of evidence of increased circulating and hepatic lipids in FEL, we performed a comprehensive analysis of the hepatic lipids of a patient with this disease. In contrast to normal qualitative characteristics and to normal or only mildly elevated concentrations of total lipids, neutral lipids, phospholipids, and neutral glycosphingolipids, this study reports a significant elevation of acidic glycosphingolipids, gangliosides ( 387 versus 147 nmole lipid-bound sialic acid/g wet weight, patient versus control). This quantitative abnormality was unusual in that it included disproportionately increased concentrations of most of the minor hepatic ganglioside species rather than either a marked increase in one single or a generalized nonspecific increase in all gangliosides, which is characteristic of known disorders exhibiting hepatic ganglioside storage. Activities of the hepatic lysosomal glycosylhydrolases which are important in ganglioside metabolism were therefore assessed by using artificial substrates. Of the enzymes tested, $\beta$-galactosidase activity alone was remarkable, being moderately reduced $(33 \%$ of that of the normal liver); no biochemical explanation for the reduction was found. The findings suggest FEL may be associated with a unique quantitative and qualitative abnormality in hepatic gangliosides, the cause of which remains to be elucidated.
\end{abstract}

\section{Abbreviations}

FEL, familial erythrophagocytic lymphohistiocytosis GLC, gas liquid chromatography

LBSA, lipid-bound sialic acid

TLC, thin layer chromatography

FEL, first described by Farquhar and Claireaux (7), is an autosomal recessive disorder which is usually rapidly fatal in early childhood $(8,38)$. Clinical findings in FEL include anorexia, hepatosplenomegaly, jaundice, thrombocytopenia, and liver dysfunction $(7,8,34,38)$. Although the pathogenesis of FEL is not understood, lymphohistiocytic infiltration with marked erythrophagocytosis in multiple organs is a uniform autopsy finding.

Recently, hyperlipidemia $(22,24,26)$ and a consistent pattern of immunodeficiency $(22,24)$, including plasma-mediated inhibition of lymphocyte proliferation in vitro, have been shown to be characteristic of FEL. The association between hyperlipidemia and plasma immunoregulatory activity in the four patients we studied suggested a possible role of abnormal lipid metabolism in the pathogenesis of this disease (22). Coupled with pathologic evidence of lipid accumulation in the liver of FEL patients $(7,38)$, the results suggested that a comprehensive study of hepatic lipids might constitute a direct approach to elucidating a potential lipid abnormality in this disease. We report herein the results of a systematic analysis of hepatic lipids and an unusual abnormality in the acidic glycosphingolipids, gangliosides, in a patient with FEL whose immunologic function we have previously studied (22).

\section{MATERIALS AND METHODS}

Total lipids. Liver samples of the 3-year-old patient and a normal, previously healthy, adult control (accident victim) were obtained within hours of death and stored at $-70^{\circ} \mathrm{C}$ until studied. Total lipids were isolated by the method of Folch et al. (9) and separated by silicic acid column chromatography (6) into three major fractions (neutral lipid, neutral glycosphingolipid, and phospholipid). The fractions were dried in vacuo, quantitated gravimetrically, and spotted on precoated 250 micron-thick TLC plates (E. Merck, Darmstadt, W. Germany) activated for $1 \mathrm{hr}$ at $110^{\circ} \mathrm{C}$. Silica Gel G plates were used for TLC of the neutral lipids and glycosphingolipids and Silica $\mathrm{Gel} \mathrm{H}$ plates (no $\mathrm{CaSO}_{4}$ binder) for TLC of the phospholipids. Neutral lipids were developed in petroleum ether:diethyl ether:acetic acid (90:10:1, v/v/v) and glycosphingolipids and phospholipids in chloroform:methanol:water $(100: 42: 6, \mathrm{v} / \mathrm{v} / \mathrm{v})$. Plates were visualized with iodine vapor for preparative TLC and with the appropriate spray reagents for qualitative TLC analysis (43).

Cholesterol was quantitated colorimetrically (1). Triglycerides were isolated by preparative TLC and subjected to methanolysis to obtain methyl esters of the individual component fatty acids (6). These were identified and quantitated by gas liquid chromatrography (GLC) (2) using methyl arachidate as the internal standard. The total molar fatty acid concentration was divided by 3 to calculate the molar triglyceride concentration. The composition of individual hepatic triglyceride species was determined by direct probe low-resolution mass spectrometry (16). Distribution of triglyceride species was calculated from the ion intensities of their characteristic mass fragmentation patterns (16). The average triglyceride molecular weight of 850 determined from these data was used to convert the molar values obtained by GLC into weight units.

Gangliosides and other glycoconjugates. A total lipid extract of liver tissue for ganglioside quantitation was obtained by the procedure of Ledeen and Yu (27). Gangliosides were measured as LBSA by the modified resorcinol assay (27). Gangliosides were purified by the Suzuki modification (45) of the method of Folch et al. (9), followed by Sephadex G-50 gel filtration to remove low molecular weight contaminants (27) before determining their thin layer chromatographic patterns. TLC was performed on precoated HPTLC Silica Gel 60 plates (E. Merck, Darmstadt, W. Germany). The plates were developed in chloroform: methanol:0.25\% aqueous $\mathrm{CaCl}_{2} \cdot 2 \mathrm{H}_{2} \mathrm{O}(60: 40: 9$, v/v/v) (27) and visualized with resorcinol- $\mathrm{HCl}$ spray reagent. Gangliosides were identified by the nomenclature of Svennerholm (48). 
Qualitative analysis of hepatic oligosaccharides in aqueous liver homogenates was performed by the method of Holmes and O'Brien (18), slightly modified to improve resolution by using silica gel $G$ plates (Redi-Plates, Fisher Scientific, Pittsburgh, PA) and by developing the plates in butanol:acetic acid:water $(3: 3: 2$, $\mathrm{v} / \mathrm{v} / \mathrm{v})$. Plates were sprayed with orcinol and resorcinol- $\mathrm{HCl}$ to visualize neutral sugars and sialic acid, respectively. Total hepatic sialic acid content was determined by GLC (27).

Lysosomal glycosylhydrolase activities. Liver samples which had been stored at $-70^{\circ} \mathrm{C}$ were homogenized in distilled water $(10 \%$ $\mathrm{w} / \mathrm{v}$ ) with three 1-min pulse intervals using a Polytron homogenizer (Brinkmann, Westbury, NY), and then freeze-thawed three times (46). The homogenates were centrifuged and the clear supernatants used immediately for the enzyme assays. Liver lysosomal glycosylhydrolase activities were determined using 4methylumbelliferyl-glycoside substrates (Koch-Light, Colnbrook, England) (53), except for neuraminidase activity, which was determined using 2(3'-methoxyphenyl)- $\alpha$ - $N$-acetylneuraminide (Boehringer-Mannheim, Indianapolis, IN) in $0.1 \mathrm{M}$ acetate buffer, pH $5.0(3,50)$. Protein concentrations were determined by the procedure of Lowry et al. (31).

\section{RESULTS}

Total lipid composition. Total lipid concentration in the FEL liver was elevated by $42 \%$ over that in the normal liver (Table 1 ). This increase was accounted for by elevations in concentrations of neutral lipids $(67 \%)$ which in turn reflected elevations in total cholesterol and triglycerides (56\% and $87 \%$, respectively). No abnormality in the distribution of individual species and no triglycerides containing unusual fatty acid components were found by mass spectroscopic analysis of the intact triglycerides. Fatty acid analysis of the isolated triglycerides showed the distribution of the component fatty acids of the triglycerides in the patient and normal control livers to be similar to each other and to the fatty acid composition previously reported for normal liver (49). The free fatty acid profile and concentration $(0.5 \%$ of the total triglyceride fatty acid concentration, Table 1) were normal (19). TLC of the FEL neutral glycosphingolipid and phospholipid fractions revealed neither qualitative nor quantitative differences from findings in the normal liver.

Gangliosides and other glycoconjugates. A 2.6-fold elevation in lipid-bound sialic acid (ganglioside) content was found in the liver of the patient, in comparison to ganglioside content of the control liver (Table 1). TLC of equal quantities of purified total gangliosides indicated a prominence, in the patient liver, of a number of gangliosides (e.g., with chromatographic mobilities of $\mathrm{G}_{\mathrm{M} 2}, \mathrm{~L}_{\mathrm{M} 1}$, $G_{M 1}, G_{T 1 b}$, and $G_{Q 1 b}$ ) (Fig. 1), which were only faintly or not visible in the TLC of the normal liver gangliosides (Fig. 1). Densitometric scanning (Fig. 2) of the same TLC plate showed that gangliosides more structurally complex than $\mathrm{G}_{\mathrm{M} 3}$ constituted $40 \%$ of the total gangliosides in the patient liver versus $14 \%$ in the control liver. The latter value was in good agreement with those of two recent studies of human liver in which gangliosides other than $\mathrm{G}_{\mathrm{M} 3}$ constituted $8.4 \%$ and $13.6 \%$ of the total $(36,42)$. Calculations of the absolute concentrations of $\mathrm{G}_{\mathrm{M} 3}$ and complex gangliosides from the sialic acid measurements and the densitometric scans indicate that the patient liver had a 1.9-fold higher concentration of $\mathrm{G}_{\mathrm{M} 3}$ and a more than 5-fold higher concentration of complex gangliosides than did the normal liver.

In contrast to this absolute increase in total gangliosides and relatively greater increase in complex gangliosides, (A) total hepatic sialic acid content was only slightly elevated above that of the control liver ( 2230 versus 1720 nmole sialic acid/g wet weight); (B) there were no qualitative or quantitative abnormalities in hepatic oligosaccharides; and (C) sialosyl-oligosaccharides were not detectable in the patient liver. These results provide evidence against the elevation in hepatic gangliosides being merely a component of a generalized increase in sialic acid-containing glycoconjugates.

Lysosomal glycosylhydrolase activities. Levels of activity of the following hepatic lysosomal enzymes were similar to those of the normal liver and within the normal range of activity previously determined by others $(3,47): \beta$ - $\mathrm{N}$-acetyl-glucosaminidase, neuraminidase, $\alpha$-fucosidase, $\alpha$-mannosidase, $\beta$-glucuronidase, and $\alpha$ galactosidase. Only $\beta$-galactosidase activity was reduced, at $33 \%$ of that of the concurrently studied control liver. The reduction in $\beta$-galactosidase activity was not due to abnormal $\mathrm{pH}$ dependence or optimum, a soluble inhibitor of enzyme activity, selective deficiency of an isoenzyme as assessed by electrophoresis, or deficiency of a heat-labile isoenzyme $(17,37)$.

\section{DISCUSSION}

The objective of this study was to determine whether a specific hepatic lipid abnormality might characterize FEL, because elevations in circulating lipids and fatty infiltration of the liver have been documented in this disease $(7,22,26,38)$. Qualitative and quantitative study of the major hepatic lipids revealed a mild increase in total lipids, essentially entirely due to elevations in the neutral lipids. At $80 \mathrm{mg} / \mathrm{g}$ wet weight, the neutral lipid content was lower than that of fatty livers (93-325 mg/g wet weight) (40), and therefore not a striking abnormality. The major components of the neutral lipid fraction (triglycerides and total cholesterol) were only mildly elevated, in contrast to the 3-100-fold degree seen in the known familial neutral lipid storage diseases (44). Combined with the fact that these lipids were qualitatively normal, significant hepatic neutral lipid storage as a primary component of FEL appears to be excluded.

We next examined the minor lipids. In these further studies, the unexpected finding was that the hepatic ganglioside concentration (387 nmole LBSA/g wet weight) was significantly above (a) that of the parallel normal control liver (147 nmole) and (b) reported normal values for adults and children (up to $254 \mathrm{nmole} \mathrm{LBSA} / \mathrm{g}$ wet weight) as determined using ganglioside isolation methods similar to those we employed $(36,42)$. The elevation in hepatic

Table 1. Quantitation of hepatic lipids

\begin{tabular}{|c|c|c|c|}
\hline & Patient' & $\begin{array}{l}\text { Normal } \\
\text { Control' }^{1}\end{array}$ & $\begin{array}{l}\text { Ranges of normal } \\
\text { literature values }\end{array}$ \\
\hline Total lipids (mg) ${ }^{2}$ & 105 & 74 & $33-85(40)$ \\
\hline Neutral lipids (mg) ${ }^{2}$ & 80 & 48 & $6.3-65(40)$ \\
\hline Total triglycerides $(\mathrm{mg})^{4}$ & 37.9 & 20.3 & $3-54(21)$ \\
\hline Free fatty acids $(\mathrm{mg})^{4}$ & 0.11 & 0.11 & $0.2(19)$ \\
\hline Neutral glycosphingolipids (mg) ${ }^{2}$ & 5 & 4 & $4.3-6.9(12)$ \\
\hline
\end{tabular}

\footnotetext{
'All values per $\mathrm{g}$ liver, wet weight.

${ }^{2}$ Gravimetric determinations, mean of duplicate samples.

${ }^{3}$ Colorimetric determinations as described in "Materials and Methods," mean of duplicate samples.

${ }^{4}$ Quantitation of component fatty acid methyl esters by gas liquid chromatography.
} 


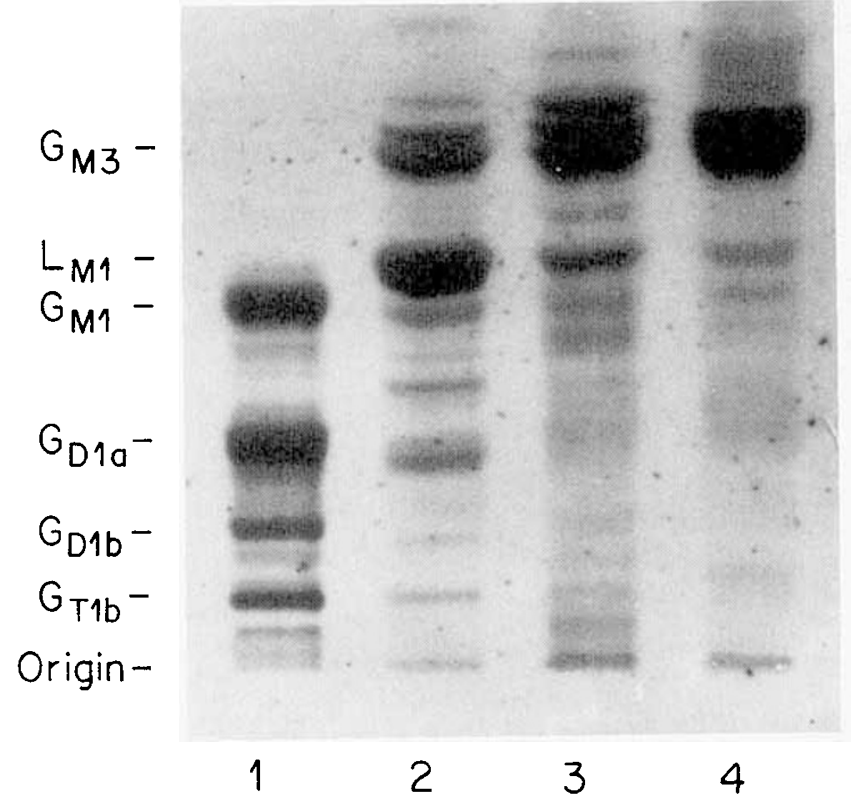

Fig. 1. Thin layer chromatography of hepatic gangliosides. Gangliosides were isolated from sources indicated, chromatographed, and visualized with resorcinol- $\mathrm{HCl}$, as described in "Materials and Methods." Lane 1 , mixed bovine brain gangliosides (10 nmole, lipid bound sialic acid, Supelco, Bellefonte, PA); Lane 2, human erythrocyte ganglioside mixture

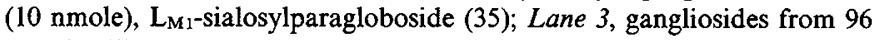
$\mathrm{mg}$ familial erythrophagocytic lymphohistiocytosis patient liver (15 nmole); Lane 4, gangliosides from $246 \mathrm{mg}$ normal liver ( $15 \mathrm{nmole}$ ). Bands above $G_{M 3}$ and at the origin were resorcinol-negative; all other bands were resorcinol-positive.
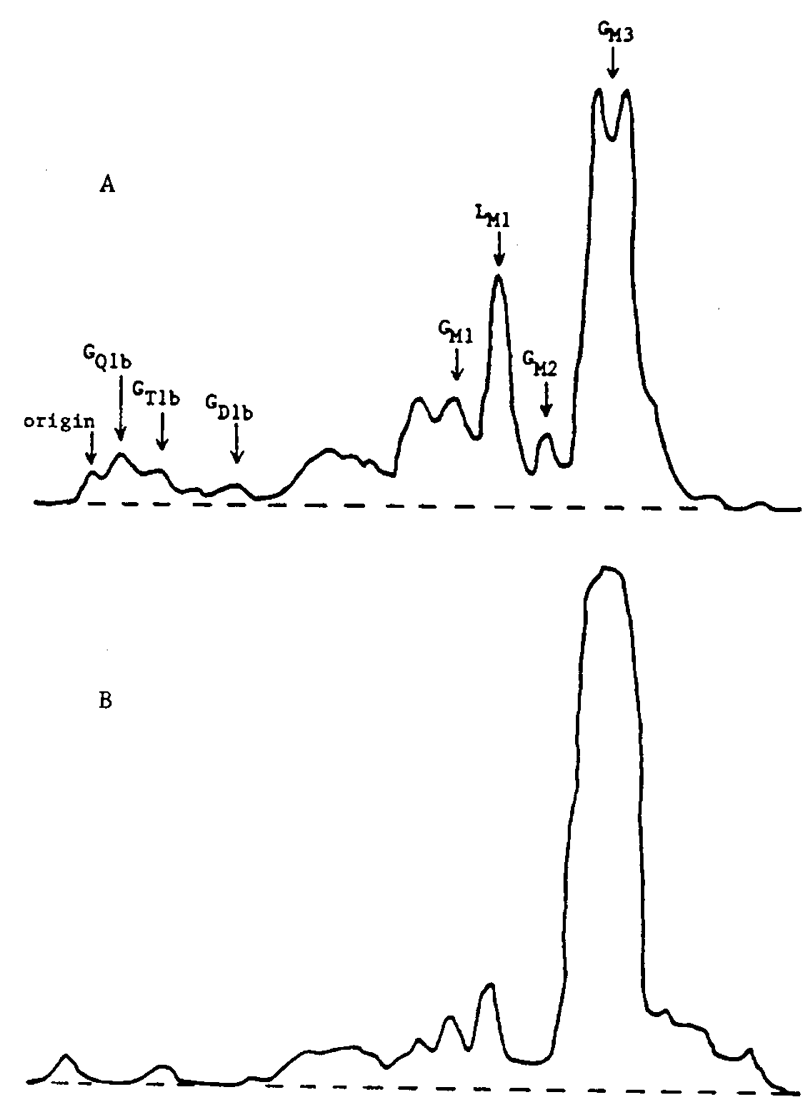

Fig. 2. Densitometric scan of the thin layer chromatography (Fig. 1) of the hepatic gangliosides of patient (panel $A$, and lane 3 in Fig. 1) and the normal control (panel B, and lane 4 in Fig. 1). Mobilities of selected ganglioside standards are indicated by arrows (lanes 1 and 2 in Figure 1). gangliosides is significant in that it is of the same magnitude (1.42.9 times normal) as that seen in $\mathrm{G}_{\mathrm{M}^{-}}$and $\mathrm{G}_{\mathrm{M} 2}$-gangliosidosis (20, 45), two glycosphingolipidoses associated with defined lysosomal enzyme defects; however, the elevations in hepatic gangliosides in these diseases $(20,37,45)$ and in $\mathrm{G}_{\mathrm{M} 3}$ gangliosidosis (41) represent marked and specific increases in a particular ganglioside. In contrast, TLC analysis of the hepatic gangliosides of our patient showed an increase in multiple gangliosides (including $\mathrm{G}_{\mathrm{M} 3}$ ), with a proportionately greater increase in some of the hepatic gangliosides of structural complexity greater than that of $G_{M 3}$. These results represent an unusual ganglioside abnormality because to our knowledge the known disorders in which hepatic ganglioside metabolism is altered are characterized by either the specific marked accumulation of a single ganglioside (as in the examples above) or a non-selective increase in all gangliosides.

A generalized increase (1.2-5.5-fold) in hepatic lipids (including gangliosides) may result even when metabolism of the lipids is not directly dependent upon the enzyme which is deficient. This may occur when lipid catabolism is impeded because of physical association of the lipids in secondary lysosomes engorged with a primary storage product (e.g., sphingomyelin in Niemann-Pick's disease) $(4,12)$, or when ganglioside catabolism is impeded because of a more distal enzymatic block (e.g., $\mathrm{G}_{\mathrm{M} 3}$ in Gaucher's disease) (10). Normal hepatic phospholipid and neutral glycosphingolipid concentrations in our patient make these explanations unlikely.

A normal concentration of oligosaccharides, which accumulate in visceral tissues in glycoprotein storage disorders (mannosidosis, fucosidosis) $(5 \mathrm{l}, 52)$ and in the known gangliosidoses $(18,35,55)$, argues against the conclusion that one of these disorders might explain the elevated hepatic gangliosides in our patient. Conditions previously defined as "sialidosis" can be excluded by the absence in this patient of hepatic sialosyl-oligosaccharides (30).

Hepatic lysosomal enzyme activities were studied to determine whether an unusual enzyme defect might account for the observed pattern of ganglioside accumulation in this patient. Although $\beta$ galactosidase activity was moderately reduced ( $33 \%$ of normal), known primary $\beta$-galactosidase deficiency associated with glycoconjugate storage diseases (Krabbe's disease, Morquio disease Type $B, G_{M 1}$ gangliosidosis) is characterized by activity of this enzyme of less than $10 \%$ of normal $(13,37,41,46)$, making it unlikely that a defect in this enzyme was responsible for the elevated hepatic ganglioside levels in our patient. Not excluded by our findings is the possibility that the observed low $\beta$-galactosidase activity represents a secondary enzyme defect, as in some cases of mucopolysaccharidosis $(17,33,47)$ and mucolipidosis $(5$, 30 ), where reduced $\beta$-galactosidase activity has been associated with a primary defect of a different lysosomal enzyme.

The other lysosomal enzyme activities, measured with fluorogenic substrates, were unremarkable; however, a potential lysosomal enzyme defect might be overlooked with these artificial substrates. For example, neuraminidase activity was not decreased in the FEL liver as measured with artificial substrate, but it has been reported that there are two distinct neuraminidase isoenzymes, only one of which can utilize gangliosides as substrates $(5$, 30 ). We have not, as yet, excluded the possibility of a deficiency of a particular isoenzyme of neuraminidase in this patient. Hepatic ganglioside accumulation could also result from the absence of a lysosomal enzyme activator protein, required for the in vivo catabolism of gangliosides $(14,15,29)$.

The elevation in hepatic gangliosides could be unrelated to an inherited enzyme defect. Certain cationic, amphiphilic drugs, such as chloroquine (11), have been shown to cause hepatic ganglioside storage, but in association with a generalized increase in most hepatic lipids, including up to 20 -fold elevations in cholesterol and neutral glycosphingolipids. Such elevations were not seen and because no toxins were found in our patient's liver, it seems unlikely that the pattern of ganglioside accumulation in the present case was toxin-related.

We also considered the possibility that the elevation in hepatic gangliosides could reflect the contribution of gangliosides present 
in cells infiltrating the liver of our patient. Erythrocytes, however, contain only one-tenth as much ganglioside per gram wet weight as normal liver (39), and lymphocytes contain mainly $\mathrm{G}_{\mathrm{M} 3}(32)$. Whether infiltrating histiocytes could have accounted for the elevated hepatic ganglioside concentration cannot be ruled out at this time because the relative contribution of macrophages and hepatocytes to total hepatic ganglioside content in normal human liver is not known (36).

As FEL is an autosomal recessive disease, we suspected that an inborn defect in ganglioside metabolism might explain the unexpected elevation of hepatic gangliosides in our patient. Because conclusive evidence for such a defect was not obtained, the pathogenesis of the ganglioside abnormality remains to be elucidated. A deficiency of a particular isoenzyme of neuraminidase would certainly be one of the most likely enzymatic lesions, and we are undertaking to determine the neuraminidase activities of various subcellular fractions of normal and the FEL liver with ganglioside substrates.

If characteristic of all FEL patients, the ganglioside abnormality, irrespective of cause, may be of clinical significance: gangliosides inhibit a number of cellular immune responses, including in vitro mitogen- and antigen-induced lymphoproliferation $(23,25$, $28,54)$, immune responses that are among those depressed in FEL $(22,24)$. Furthermore, because the plasma of patients with FEL inhibits these same immune responses (22), while removal of patient plasma in vivo by repeated blood exchange was accompanied by (A) abrogation of the plasma inhibitory activity, (B) reversal of depressed cellular immune responses, and (C) transient clinical improvement (24), it should be of interest to determine whether the hepatic ganglioside abnormality is also reflected in the plasma of these patients.

\section{REFERENCES AND NOTES}

1. Abell, L. L., Levy, B. B., Brodie, B. B., and Kendall, F. E.: A simplified method for the estimation of total cholesterol in serum and demonstration of its specificity. J. Biol. Chem., 195: 357 (1952).

2. Ackman, R. G.: Gas-liquid chromatography of fatty acids and esters. Methods Enzymol., 14: 329 (1969).

3. Alhadeff, J. A. and Wolfe, S.: Characterization of human liver (4-methylumbelliferyl- $\alpha-D-N$-acetylneuraminic acid) neuraminidase activity. Int. J. Biochem.. 13: 975 (1981).

4. Brunngraber, E. G.. Berra, B., and Zambotti, V.: Altered levels of tissue glycoproteins, gangliosides, glycosaminoglycans and lipids in Niemann-Pick's disease. Clin. Chim. Acta, 48: 173 (1973).

5. Cantz, M. and Messer, H.: Oligosaccharide and ganglioside neuraminidase activities of mucolipidosis I (sialidosis) and mucolipidosis II (I-cell disease) fibroblasts. Eur. J. Biochem., 97: 113 (1979).

6. Esselman. W. J., Laine, R. A., and Sweeley, C. C.: Isolation and characterization of glycosphingolipids. Methods Enzymol., 28: 140 (1972).

7. Farquhar, J. W. and Claireaux. A. E.: Familial haemophagocytic reticulosis. Arch. Dis. Child., 27: 519 (1952).

8. Farquhar, J. W.. MacGregor, A. R., and Richmond, J.: Familial erythrophagocytic reticulosis. Br. Med. J., II: 1561 (1958).

9. Folch. J., Lees, M. and Sloane-Stanley, G. H.: A simple method for the isolation and purification of total lipides from animal tissues. J. Biol. Chem., 226: 497 (1957).

10. Fredrickson. D. S. and Sloan H. R.: Glucosyl ceramide lipidoses: Gaucher's disease, In: J. B. Stanbury, J. B., Wyngaarden, and D. S. Fredrickson: The Metabolic Basis of Inherited Disease, p. 730. (McGraw-Hill. New York, N.Y.. 1972).

11. Fredman, P.. Klinghardt, G. W., Nilsson, O., and Svennerholm, L.: Lipid accumulation in liver, spleen. lung. and kidneys of miniature pigs after chloroquine treatment. Biochem. J., 201: 581 (1982).

12. Gilbert, E. F.. Callahan. J., Viseskul, C., and Opitz, J. M.: Niemann-Pick Disease Type C. Pathological, histochemical, ultrastructural and biochemical studies. Eur. J. Pediatr., 136: 263 (1981).

13. Groebe, H., Krins, M., Schmidberger, H., Von Figura, K., Harzer, K., Kresse, H.. Paschke, E., Sewell, A., and Ullrich. K.: Morquio Syndrome (Mucopolysaccharidosis IV B) associated with $\beta$-galactosidase deficiency: 2 cases. Am. J. Hum. Genet., 32: 258 (1980).

14. Hechtman. P.: Characterization of an activating factor required for hydrolysis of $\mathrm{G}_{\mathrm{M}:}$ ganglioside catalyzed by hexosaminidase A. Can. J. Biochem., 55: 315 (1977).

15. Hechtman, P.. Gordon, B. A.. and Ng Ying Kin. N. M. K.: Deficiency of the hexosaminidase $A$ activator protein in a case of $G_{M 2}$ gangliosidosis; variant AB. Pediatr. Res., 16: 217 (1982).

16. Hites. R. A.: Mass spectrometry of triglycerides. Methods Enzymol.. 35: 348 (1975).

17. Ho. M. W. and O'Brien. J. S.: Hurler's syndrome: deficiency of a specific beta galactosidase isoenzyme. Science, 165: 611 (1969).
18. Holmes, E. W. and O'Brien, J. S.: Separation of glycoprotein-derived oligosaccharides by thin-layer chromatography. Anal. Biochem., 93: 167 (1979).

19. Konno, T., Fugii, M., Watanuki, T., and Koizumi, K.: Wolman's disease: the first case in Japan. Tohoku J. Exp. Med., 90: 375 (1966).

20. Kudoh, $\Upsilon$., Orii, $\Upsilon$., Nakao, $\Upsilon$., and Sakagami, $\Upsilon$.: Three cases of $\mathrm{G}_{M 1}$-gangliosidosis. Clin. Chim. Acta., 70: 277 (1976).

21. Kwiterovich, P. O. Jr., Sloan, H. R., and Fredrickson, D. S.: Glycolipids and other lipid constituents of normal human liver. J. Lipid Res., 11: 322 (1970).

22. Ladisch, S., Holiman, B., Poplack, D. G., and Blaese, R. M.: Immunodeficiency in familial erythrophagocytic lymphohistiocytosis. Lancet I: 581 (1978).

23. Ladisch, S., Wong, C., Ulsh, L., and DeLay, A-M.: Suppression of antigen specific lymphocyte blastogenic responses by tumor cell-derived gangliosides. Clin. Res., 29: 528A (1981).

24. Ladisch, S., Ho, W., Matheson, D., Pilkington, R., and Hartman, G.: Immunologic and clinical effects of repeated blood exchange in familial erythrophagocytic lymphohistiocytosis. Blood, 60: 814 (1982).

25. Ladisch, S., Ulsh, L., Gillard, B., and Wong, C.: Inhibition of lymphocyte proliferation by gangliosides is mediated by an effect on adherent cells. 15th International Leucocyte Culture Conference, Dec., 1982. Immunobiology, 163: 120 (1982).

26. Landrieu, P. and Choulot, J.-J.: Reticulose hemophagocytaire avec hypertriglyceridemie. Arch. Fr. Pediatr., 33: 497 (1976).

27. Ledeen, R. W. and Yu, R. K.: Gangliosides: structure, isolation and analysis. Methods Enzymol., 83: 139 (1982).

28. Lengle, E. E.: Increased levels of lipid-bound sialic acid in thymic lymphocytes and plasma from leukemic AKR/J mice. J. Natl. Cancer Inst., 62: 1565 (1979).

29. Li, S.-C., Nakamura, T., Ogamo, A., and Li, Y.-T.: Evidence for the presence of two separate protein activators for the enzymic hydrolysis of $G_{M 1}$ and $G_{M 2}$ gangliosides. J. Biol. Chem., 254: 10592 (1979).

30. Lowden, A. J. and O'Brien, J. S.: Sialidosis: a review of human neuraminidase deficiency. Am. J. Hum. Genet., 3I: 1 (1979).

31. Lowry, O. H., Rosebrough, N. J., Farr, A. L., and Randall, R. J.: Protein measurement with the Folin phenol reagent. J. Biol. Chem., 193: 265 (1951).

32. Macher, B. A., and Klock, J. C., Fukuda, M. N., and Fukuda, M.: Isolation and structural characterization of human lymphocyte and neutrophil gangliosides. J. Biol. Chem., 256: 1968 (1981).

33. McKusick, V. A., Neufeld, E. F., and Kelly, T. E.: The mucopolysaccharide storage diseases. In: J. B. Stanbury, J. B. Wyngaarden, and D. S. Fredrickson: The Metabolic Basis of Inherited Disease, p. 1282. (McGraw-Hill, New York, NY, 1978)

34. Mozziconacci, P., Nezelof, C., Attal, C., Girard, F., Pham-Huu-Trung, Weil, J., Desbuquois, B., and Gadot, M.: La Lympho-histiocytose familiale. Arch. Fr Pediatr., 22: 385 (1965)

35. Ng Ying Kin, N. M. K. and Wolfe, L. S.: Oligosaccharides accumulating in the

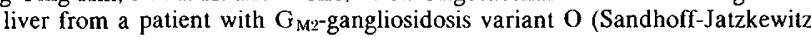
Disease). Biochem. Biophy. Res. Commun., 59: 837 (1974).

36. Nilsson, O. and Svennerholm, L.: Characterization and quantitative determination of gangliosides and neutral glycosphingolipids in human liver. J. Lipid Res., 23: 327 (1982).

37. O'Brien, J. S.: The gangliosidoses. In: J. B. Stanbury, J. B. Wyngaarden, and D. S. Fredrickson: The Metabolic Basis of Inherited Disease, p. 841. (McGrawHill, New York, N.Y., 1978).

38. Perry, M. C., Harrison, Jr., E. G., Burgert, Jr., E. O., and Gilchrist, G. S.: Familial Erythrophagocytic Lymphohistiocytosis. Cancer. 38: 209 (1976).

39. Portoukalian, J., Zwingelstein, G., Abdul-Malak, N., and Doré, J. F.: Alteration of gangliosides in plasma and red cells of humans bearing melanoma tumors. Biochem. Biophys. Res. Commun., 85: 916 (1978)

40. Ralli, E. P. Rubin, S. H., and Rinzler, S.: The liver lipids in normal human livers and in cases of cirrhosis and fatty infiltration of the liver. J. Clin. Invest., 20 93 (1941).

41. Sandhoff, $\mathrm{K}$. and Christomanou, H.: Biochemistry and genetics of gangliosidoses. Hum. Genet., 50: 107 (1979).

42. Seyfried, T. N., Ando, S., and Yu, R. K.: Isolation and characterization of human liver hematoside. J. Lipid Res., 19: 538 (1978).

43. Skipski. V. P. and Barclay, M.: Thin-layer chromatography of lipids. Methods Enzymol. 14: 530 (1969)

44. Sloan, H. R. and Fredrickson, D. S.: Rare familial disease with neutral lipid storage: Wolman's disease, cholesterol ester storage disease, and cerebrotendinous xanthomatosis. In: J. B. Stanbury, J. B. Wyngaarden, and D. S. Fredrickson: The Metabolic Basis of Inherited Disease, p. 808. (McGraw-Hill. New York, N.Y.. 1972).

45. Suzuki, $K_{\text {.: }}$ Cerebral $G_{M_{1}}$-gangliosidosis:chemical pathology of visceral organs. Science, 159: 1471 (1968).

46. Suzuki, K.: Galactocerebroside $\beta$-galactosidase in Krabbe's Globoid Cell Leukodystrophy. Methods Enzymol., 28: 839 (1972).

47. Suzuki, Y., Fukuoka, K., Wey, J. J., and Handa, S.: $\beta$-Galactosidase in mucopolysaccharidoses and mucolipidoses. Deficiency of $\mathrm{G}_{\mathrm{M}} \beta$-galactosidase in liver and leukocytes. Clin. Chim. Acta, 75: 91 (1977).

48. Svennerholm, L.: Chromatographic separation of human brain gangliosides. J. Neurochem.. 10: 613 (1963).

49. Takahashi, Y. and Tanaka, K.: Gas-chromatographic analysis of the fatty acid composition of human fatty liver. J. Biochem.. 49: 713 (1961).

50. Thomas, G. H., Tipton, R. E., Chien, L. T., Reynolds, L. W., and Miller, C. S. Sialidase ( $\alpha-N$-acetyl neuraminidase) deficiency: the enzyme defect in an adult with macular cherry-red spots and myoclonus without dementia. Clin. Genet., 13: 369 (1978).

51. Tsay, G. C., Dawson. G.. and Matalon. R.: Glycopeptide storage in skin fibroblasts cultured from a patient with $\alpha$-mannosidase deficiency. J. Clin. Invest., 56: 711 (1975). 
52. Tsay, G. C., Dawson, G., and Sung, S.-S. J.: Structure of the accumulating oligosaccharide in fucosidosis. J. Biol. Chem., 251: 5852 (1976).

53. Wenger, D. A., Sattler, M., Clark, C., and Wharton, C.: I-Cell Disease: Activities of lysosomal enzymes toward natural and synthetic substrates. Life Sci., 19: 413 (1976).

54. Whisler, R. L. and Yates, A. J.: Regulation of lymphocyte responses by human gangliosides. J. Immunol., 125: 2106 (1980).

55. Wolfe, L. S., Senior, R. G., and Ng Ying Kin, N. M. K.: The structures of oligosaccharides accumulating in the liver of $\mathrm{G}_{M 1}$-gangliosidosis, Type I. $\mathbf{J}$. Biol. Chem., 249: 1828 (1974).

56. Requests for reprints should be addressed to: Stephan Ladisch, M.D., Department of Pediatrics, Division of Hematology-Oncology, UCLA School of Medicine,

Copyright @ 1983 International Pediatric Research Foundation, Inc. 0031-3998/83/1705-0413\$02.00/0
Los Angeles, CA 90024.

57. This study was supported in part by Grants CA27701 and AM 12434 from the National Institutes of Health, and aided by Clinical Research Grant 6-268 from the March of Dimes Birth Defects Foundation. In part, the work was performed in partial fulfillment of the requirements fro the degree of Ph.D. (C.G.W.), Department of Biochemistry, Michigan State University, 1980.

Stephan Ladisch is the recipient of a Research Career Development Award IK04 CA00821 from the National Cancer Institute. We thank Ellen Potter and Donna Lopez for preparation of the manuscript.

58. Received for publication June 22, 1982.

59. Accepted for publication January 13, 1983. 